\title{
Analysis of the Stability of the Riemann Problem for a Simplified Model in Magnetogasdynamics
}

\author{
Yujin Liu and Wenhua Sun \\ School of Science, Shandong University of Technology, Zibo, Shandong 255049, China \\ Correspondence should be addressed to Yujin Liu; yjliu98@126.com
}

Received 19 January 2016; Revised 22 April 2016; Accepted 3 May 2016

Academic Editor: Ciprian G. Gal

Copyright ( 2016 Y. Liu and W. Sun. This is an open access article distributed under the Creative Commons Attribution License, which permits unrestricted use, distribution, and reproduction in any medium, provided the original work is properly cited.

\begin{abstract}
The generalized Riemann problem for a simplified model of one-dimensional ideal gas in magnetogasdynamics in a neighborhood of the origin $(t>0)$ in the $(x, t)$ plane is considered. According to the different cases of the corresponding Riemann solutions, we construct the perturbed solutions uniquely with the characteristic method. We find that, for some case, the contact discontinuity appears after perturbation while there is no contact discontinuity of the corresponding Riemann solution. For most cases, the Riemann solutions are stable and the perturbation can not affect the corresponding Riemann solutions. While, for some few cases, the forward (backward) rarefaction wave can be transformed into the forward (backward) shock wave which shows that the Riemann solutions are unstable under such local small perturbations of the Riemann initial data.
\end{abstract}

\section{Introduction}

Magnetogasdynamics plays an important role when we study engineering physics and many other aspects (see [1-4] and the references cited therein) and is important for the hyperbolic theory. An inviscid and perfectly conducting compressible fluid, subject to a transverse magnetic field, is given by

$$
\begin{aligned}
& \rho_{t}+\operatorname{div}(\rho u)=0, \\
& (\rho u)_{t}+\operatorname{div}(\rho u \otimes u+p I)-\mu \operatorname{rot} H \times H=0, \\
& \left(\rho E+\frac{1}{2} \mu H^{2}\right)_{t}+\operatorname{div}(\rho u E+u p-\mu(u \times H) \times H) \\
& \quad=0 \\
& H_{t}-\operatorname{rot}(u \times H)=0 \\
& \operatorname{div} H=0 \\
& p=f(\rho, S),
\end{aligned}
$$

where $\rho \geq 0, p, u, S, B \geq 0, \mu, e$, and $E=e+u^{2} / 2$ denote the density, pressure, velocity, specific entropy, transverse magnetic field, magnetic permeability, specific internal energy, and specific total energy, respectively. $u=\left(u_{1}, u_{2}, u_{3}\right)$ is the velocity of the fluid in the direction of $\left(x_{1}, x_{2}, x_{3}\right)$, $H=\left(H_{1}, H_{2}, H_{3}\right)$ is the magnetic field in the direction of $\left(x_{1}, x_{2}, x_{3}\right)$ and $H=\mu B$.

Raja Sekhar and Sharma [5] studied the one-dimensional unsteady flow of an isentropic, inviscid, and perfectly conducting compressible fluid, subject to a transverse magnetic field:

$$
\begin{aligned}
\rho_{t}+(\rho u)_{x} & =0, \\
(\rho u)_{t}+\left(\rho u^{2}+p+\frac{B^{2}}{2}\right)_{x} & =0,
\end{aligned}
$$

where $B=k \rho$ and $k$ are positive constant $[2,5]$. They constructed the Riemann solutions and discussed the interactions of the elementary waves.

Shen [6] studied the Riemann problem for (2) further and found that the Riemann solutions converge to the corresponding Riemann solutions of the transport equations by letting both the pressure and the magnetic field vanish. 
$\mathrm{Hu}$ and Sheng [2] investigated the Riemann problem of the one-dimensional inviscid and perfectly conducting compressible fluid

$$
\begin{array}{r}
\tau_{t}-u_{x}=0 \\
u_{t}+\left(p+\frac{B^{2}}{2 \mu}\right)_{x}=0 \\
\left(E+\frac{B^{2} \tau}{2 \mu}\right)_{t}+\left(p u+\frac{B^{2} u}{2 \mu}\right)_{x}=0
\end{array}
$$

with the initial data

$$
(\tau, p, u)(x, 0)=\left(\tau^{ \pm}, p^{ \pm}, u^{ \pm}\right), \quad \pm x>0,
$$

under the assumption $B=k \rho$, where $\tau^{ \pm}, p^{ \pm}, u^{ \pm}$are arbitrary constants and $\tau>0$ is the specific volume. They obtained constructively the unique solution of the Riemann problem with the characteristic method.

In [7], we removed the above assumption $B=k \rho$ and mainly consider the Riemann problem of the onedimensional unsteady flow of an isentropic, inviscid, and perfectly conducting compressible fluid

$$
\begin{aligned}
\rho_{t}+(\rho u)_{x} & =0, \\
(\rho u)_{t}+\left(\rho u^{2}+p+\frac{B^{2}}{2}\right)_{x} & =0, \\
(B)_{t}+(B u)_{x} & =0,
\end{aligned}
$$

where $p=A \rho^{\gamma}$ holds for the polytropic gas, $A$ is a positive constant, and $\gamma$ is the adiabatic constant.

Since the governing equations are nonlinear and complex, the results for magnetogasdynamics are less than the conventional gas dynamics while many results are similar to each other $[1,8]$. However the contact discontinuity is very different from each other. Unlike the conventional gas dynamics, where the image of the contact discontinuity in the space $(\tau, p, u)$ is a straight line parallel to the $\tau$-axis and the projection on the plane $(p, u)$ is a point, the contact discontinuity is a plane curve in $(\tau, p, u)$ and the projection on $(p, u)$ is a straight line parallel to the $p$-axis which causes the fact that the Riemann solutions are more complicated than that of the conventional gas dynamics.

The generalized Riemann problem of the conventional gas dynamics is studied by many authors (see [9-14], etc.).

In this paper, we mainly consider the generalized Riemann problem of (3) with the initial data

$$
(\tau, p, u)(x, 0)=\left(\tau_{0}^{ \pm}, p_{0}^{ \pm}, u_{0}^{ \pm}\right)(x), \quad \pm x>0,
$$

where $\tau_{0}^{ \pm}(x), p_{0}^{ \pm}(x)$, and $u_{0}^{ \pm}(x)$ are arbitrary smooth functions satisfying

$$
\lim _{x \rightarrow 0 \pm}\left(\tau_{0}^{ \pm}, p_{0}^{ \pm}, u_{0}^{ \pm}\right)(x)=\left(\tau^{ \pm}, p^{ \pm}, u^{ \pm}\right)
$$

Problem (3) and (6) may be regarded as a perturbation of the corresponding Riemann problem (3) and (4). Naturally, we wonder if the solutions of (3) and (6) are similar to the corresponding Riemann solutions of (3) and (4) in the neighborhood of the origin. According to the different cases of the corresponding Riemann solutions of (3) and (4), we construct the perturbed solutions with the characteristic method. We find that the contact discontinuity appears after perturbation while the corresponding Riemann solution has no contact discontinuity for some case. For most cases, the Riemann solutions are stable and the perturbation can not affect the corresponding Riemann solutions. While, for some few cases, the forward (backward) rarefaction wave can be transformed into the forward (backward) shock wave which shows that the Riemann solutions are unstable under such small perturbations of the Riemann initial data.

The paper is organized as follows. Section 2 gives the elementary waves and the Riemann solutions of (3) and (4) for our later discussions. According to different cases of the corresponding Riemann solutions of (3) and (4), in Section 3, we construct case by case the perturbed Riemann solutions of (3) and (6).

\section{Solutions of the Riemann Problem (3) and (4)}

First, we give briefly the elementary waves and the Riemann solutions for system (3) and list some notations which are used in subsequent sections. We refer readers to [2] for details.

There are three eigenvalues of (3) which are $\lambda_{-}=-((p-$ $\left.\left.e_{p} B B_{\tau} / \mu+e_{\tau}\right) / e_{p}\right)^{1 / 2}, \lambda_{0}=0$, and $\lambda_{+}=\left(\left(p-e_{p} B B_{\tau} / \mu+\right.\right.$ $\left.\left.e_{\tau}\right) / e_{p}\right)^{1 / 2}$. If $e_{p}>0$ and $e_{\tau}+p>0$, they are real and distinct; thus (3) is a strictly hyperbolic system. It is easily shown that the characteristic fields $\lambda_{ \pm}$are genuinely nonlinear and the characteristic field $\lambda_{0}$ is linearly degenerate.

For the polytropic gas, the forward or backward rarefaction wave in the $(\tau, p, u)$ space consisting of all the states which can be connected to the state $Q_{0}\left(\tau_{0}, p_{0}, u_{0}\right)$ on the right is given by

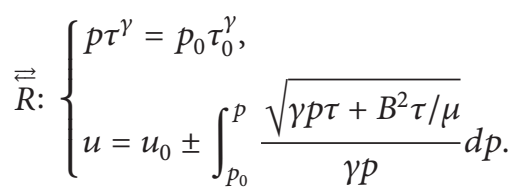

The contact discontinuity can be expressed as

$$
J:[u]=\left[p+\frac{B^{2}}{2 \mu}\right]=\sigma=0
$$

and it is easy to see that $J$ is a curve with $u=$ Const. in the $(\tau, p, u)$ space and the projection on the $(p, u)$ plane is a straight line parallel to the $p$-axis. 
The forward or backward shock wave in the $(\tau, p, u)$ space consisting of all the states which can be connected to the state $Q_{0}\left(\tau_{0}, p_{0}, u_{0}\right)$ on the right is given by

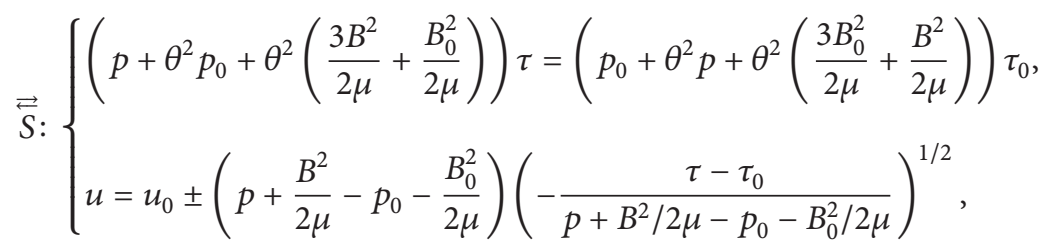

where $\theta^{2}=(\gamma-1) /(\gamma+1)$ and $B_{0}=k / \tau_{0}$.

For convenience and conciseness, denote the projection of $\underset{R}{\stackrel{\rightleftarrows}{(S}})$ on the $(\tau, p)$ plane and $(p, u)$ plane by $R_{u}\left(S_{u}\right)$ and $\vec{R}_{\tau}\left(\vec{S}_{\tau}\right)$, respectively. Denote the contact discontinuity $J$ by $\hat{J}$ when $p_{l}<p_{r}, \tau_{l}<\tau_{r}$ and $\vec{J}$ when $p_{l}>p_{r}, \tau_{l}>\tau_{r}$.

In order to construct the Riemann problem of (3) and (4), we denote $\overleftarrow{W}_{-\tau}\left(Q_{-\tau}\right)=\overleftarrow{R}_{-\tau}\left(Q_{-\tau}\right) \cup \bar{S}_{-\tau}\left(Q_{-\tau}\right)$ and $\vec{W}_{+\tau}\left(Q_{+\tau}\right)=\vec{R}_{+\tau}\left(Q_{+\tau}\right) \cup \vec{S}_{+\tau}\left(Q_{+\tau}\right)$, where $Q_{-\tau}$ and $Q_{+\tau}$ are, respectively, the projections of $Q_{-}$and $Q_{+}$on the plane $(p, u)$. Draw $\overleftarrow{W}_{-\tau}\left(Q_{-\tau}\right)$ from $Q_{-\tau}$ and $\vec{W}_{+\tau}\left(Q_{+\tau}\right)$ from $Q_{+\tau}$ in the plane $(p, u)$, respectively. According to the properties of $\overleftarrow{W}_{-\tau}\left(Q_{-\tau}\right)$ and $\vec{W}_{+\tau}\left(Q_{+\tau}\right)$, they intersect with each other at most once. Therefore, there are five cases: $\overleftarrow{W}_{-\tau}\left(Q_{-\tau}\right) \cap$ $\vec{W}_{+\tau}\left(Q_{+\tau}\right)=\left(\overleftarrow{R}_{-\tau}\left(Q_{-\tau}\right) \cap \vec{R}_{+\tau}\left(Q_{+\tau}\right)\right)$ or $\left(\overleftarrow{S}_{-\tau}\left(Q_{-\tau}\right) \cap \vec{R}_{+\tau}\left(Q_{+\tau}\right)\right)$ or $\left(\overleftarrow{R}_{-\tau}\left(Q_{-\tau}\right) \cap \vec{S}_{+\tau}\left(Q_{+\tau}\right)\right)$ or $\left(\hat{S}_{-\tau}\left(Q_{-\tau}\right) \cap \vec{S}_{+\tau}\left(Q_{+\tau}\right)\right)$ or $\varnothing$.

For the last case, we easily know there is a vacuum solution. In what follows, we just need to consider the first case since the other cases can be studied similarly.

Suppose $\overleftarrow{W}_{-\tau}\left(Q_{-\tau}\right) \cap \vec{W}_{+\tau}\left(Q_{+\tau}\right)=\overleftarrow{R}_{-\tau}\left(Q_{-\tau}\right) \cap \vec{R}_{+\tau}\left(Q_{+\tau}\right)=$ $\left\{Q_{* \tau}\right\}$ (see Figure 1); we know there exists $\left(p_{*}, u_{*}\right)$ satisfying

$$
\begin{aligned}
& u_{*}=u_{-}+\int_{p_{-}}^{p_{*}} \frac{\sqrt{\gamma p \tau+k B / \mu}}{\gamma p} d p, \quad p \tau^{\gamma}=p_{-} \tau_{-}^{\gamma}, \\
& u_{*}=u_{+}-\int_{p_{+}}^{p_{*}} \frac{\sqrt{\gamma p \tau+k B / \mu}}{\gamma p} d p, \quad p \tau^{\gamma}=p_{+} \tau_{+}^{\gamma} .
\end{aligned}
$$

Denote

$$
\begin{aligned}
& f_{1}\left(p_{1}\right):=u_{-}+\int_{p_{1}}^{p_{-}} \frac{\sqrt{\gamma p \tau+k B(\tau) / \mu}}{\gamma p} d p, \\
& p \tau^{\gamma}=p_{-} \tau_{-}^{\gamma}, p_{1} \in\left[0, p_{-}\right], \\
& f_{2}\left(p_{2}\right):=u_{+}-\int_{p_{2}}^{p_{+}} \frac{\sqrt{\gamma p \tau+k B(\tau) / \mu}}{\gamma p} d p, \\
& g_{1}\left(p_{1}\right):=p_{1}+\frac{B_{1}^{2}\left(\tau_{1}\right)}{2 \mu}, \\
& g_{2}\left(p_{2}\right):=p_{-} \tau_{-}^{\gamma}, p_{2} \in\left[0, p_{+}\right], \\
& 2 \mu
\end{aligned}
$$

where $\tau_{1}$ satisfies

$$
\begin{aligned}
& \left(p_{1}+\theta^{2} p_{-}+\theta^{2}\left(\frac{3 B_{1}^{2}\left(\tau_{1}\right)}{2 \mu}+\frac{B_{-}^{2}}{2 \mu}\right)\right) \tau_{1} \\
& =\left(p_{-}+\theta^{2} p_{1}+\theta^{2}\left(\frac{3 B_{-}^{2}\left(\tau_{1}\right)}{2 \mu}+\frac{B_{1}^{2}}{2 \mu}\right)\right) \tau_{-},
\end{aligned}
$$

$p_{1} \tau_{1}^{\gamma}=p_{-} \tau_{-}^{\gamma}, \quad p_{1} \leq p_{-}$

$$
p_{1}>p_{-},
$$

and $\tau_{2}$ satisfies

$$
\begin{aligned}
& \left(p_{2}+\theta^{2} p_{+}+\theta^{2}\left(\frac{3 B_{2}^{2}\left(\tau_{2}\right)}{2 \mu}+\frac{B_{+}^{2}}{2 \mu}\right)\right) \tau_{2} \\
& =\left(p_{+}+\theta^{2} p_{2}+\theta^{2}\left(\frac{3 B_{+}^{2}\left(\tau_{2}\right)}{2 \mu}+\frac{B_{2}^{2}}{2 \mu}\right)\right) \tau_{+},
\end{aligned}
$$

$p_{2}>p_{+}$,

$p_{2} \tau_{2}^{\gamma}=p_{+} \tau_{+}^{\gamma}, \quad p_{2} \leq p_{+}$.

Denote

$h_{1}\left(p_{1}\right):=u_{-}$

$-\sqrt{\left(p_{1}+\frac{B_{1}^{2}\left(\tau_{1}\right)}{2 \mu}-p_{-}-\frac{B_{-}^{2}}{2 \mu}\right)\left(\tau_{-}-\tau_{1}\right)}$,

$h_{2}\left(p_{2}\right):=u_{+}$

$$
+\sqrt{\left(p_{2}+\frac{B_{2}^{2}\left(\tau_{2}\right)}{2 \mu}-p_{+}-\frac{B_{+}^{2}}{2 \mu}\right)\left(\tau_{+}-\tau_{2}\right)},
$$

where $\tau_{1}$ satisfies the first equation of (13) and $\tau_{2}$ satisfies the first equation of (14).

Let

$$
\begin{aligned}
& f_{1}\left(p_{1}\right)=f_{2}\left(p_{2}\right), \\
& g_{1}\left(p_{1}\right)=g_{2}\left(p_{2}\right), \\
& f_{1}\left(p_{1}\right)=h_{2}\left(p_{2}\right), \\
& g_{1}\left(p_{1}\right)=g_{2}\left(p_{2}\right), \\
& h_{1}\left(p_{1}\right)=f_{2}\left(p_{2}\right), \\
& g_{1}\left(p_{1}\right)=g_{2}\left(p_{2}\right) .
\end{aligned}
$$




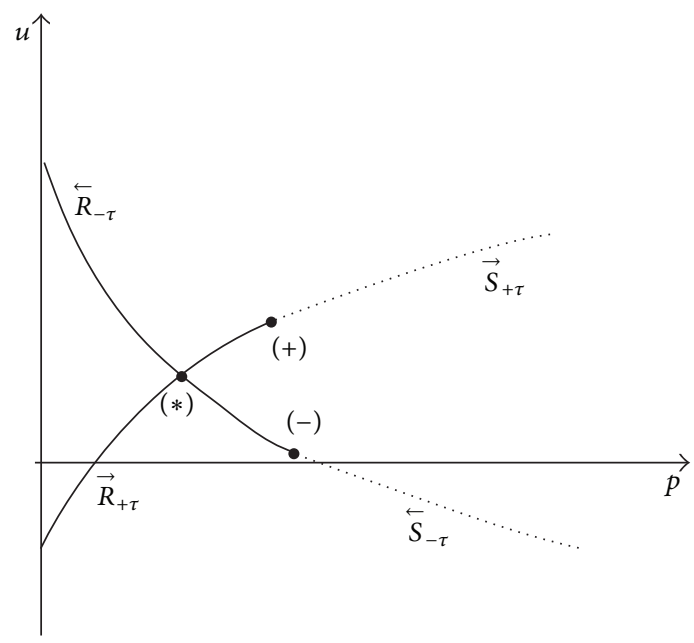

FIGURE 1: Elementary waves in the $(u, p)$ plane.

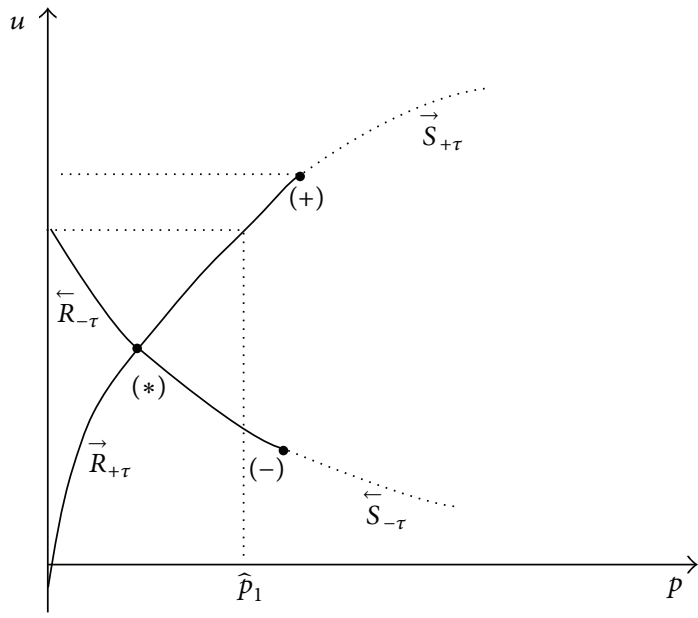

Figure 2: $f_{1}(0) \leq u_{+}=f_{2}\left(p_{+}\right)$.

In [2], the authors proved that only one of the above three equations, (16), (17), and (18), is solvable and the solution is unique which implies that there exists a unique contact discontinuity $J$ joining the two states which are located on $\stackrel{\rightleftarrows}{R}$ and $\stackrel{\leftrightarrows}{S}$, respectively.

Next we list case by case the Riemann solutions of (3) and (4) and refer readers to [2] for details.

Case $1\left(p_{-} \tau_{-}^{\gamma}=p_{+} \tau_{+}^{\gamma}\right)$. It holds that $g_{1}\left(p_{*}\right)=g_{2}\left(p_{*}\right)$ and $p_{1}=$ $p_{2}=p_{*}$ is the solution of (16). Thus, the Riemann solution is $\overleftarrow{R}+\vec{R}$; here the symbol "+" means "followed by." We notice that for this case there is no contact discontinuity.

Case $2\left(p_{-} \tau_{-}^{\gamma}<p_{+} \tau_{+}^{\gamma}\right)$.

Subcase $2.1\left(f_{1}(0) \leq u_{+}\right.$(see Figure 2$)$ ). It is obvious that there exists a point $\hat{p}_{1} \in\left(p_{*}, p_{+}\right)$such that $f_{1}(0)=f_{2}\left(\widehat{p}_{1}\right)$ and $g_{1}(0)<g_{2}\left(\widehat{p}_{1}\right)$. It follows that the Riemann solution is $\overleftarrow{R}+\stackrel{\zeta}{J}$ $+\vec{R}$.

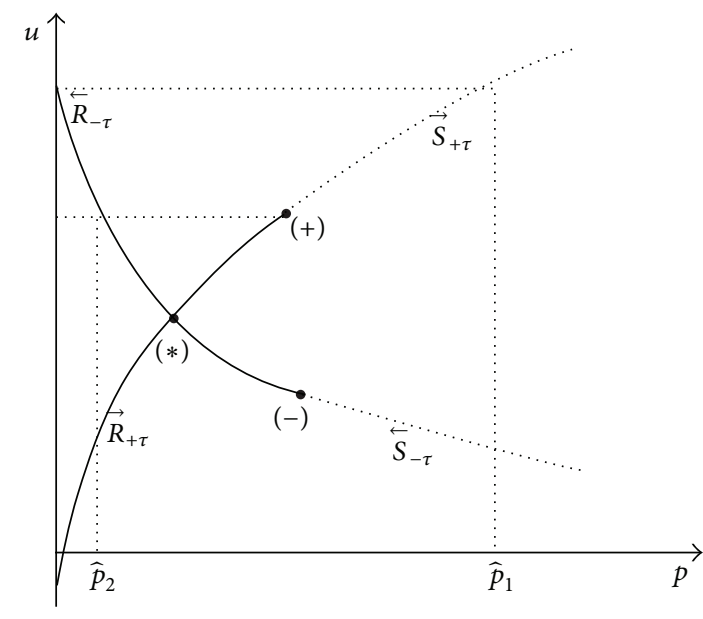

Figure 3: $f_{1}(0)>u_{+}=f_{2}\left(p_{+}\right)$.

Subcase $2.2\left(f_{1}(0)>u_{+}\right.$(see Figure 3$\left.)\right)$. Since there exists a point $\widehat{p}_{2} \in\left(0, p_{*}\right)$ such that $f_{1}\left(\widehat{p}_{2}\right)=u_{+}$, we divide it into two subcases.

Subcase 2.2.1. If $g_{1}\left(\hat{p}_{2}\right) \leq g_{2}\left(p_{+}\right)$, due to $h_{2}^{\prime}\left(p_{2}\right)>0$ and $h_{2}\left(p_{+}\right)=f_{2}\left(p_{+}\right)<f_{1}(0)$, considering that the curve $h_{2}\left(p_{2}\right)$ has no asymptote, then there exists a point $\widehat{p}_{*} \in\left(p_{+},+\infty\right)$ such that $h_{2}\left(\widehat{p}_{*}\right)=f_{1}(0)$. From the smoothness of the curve and $g_{2}\left(\widehat{p}_{*}\right)>g_{2}(0)$, there exists $\left(\bar{p}_{1}, \bar{p}_{2}\right) \in\left(0, \hat{p}_{2}\right) \times\left(p_{+}, \widehat{p}_{*}\right)$ such that $f_{1}\left(\bar{p}_{1}\right)=h_{2}\left(\bar{p}_{2}\right)$ and $g_{1}\left(\bar{p}_{1}\right)=g_{2}\left(\bar{p}_{2}\right)$. Therefore, $\left(\bar{p}_{1}, \bar{p}_{2}\right)$ is the point which we seek for and the Riemann solution is $\overleftarrow{R}+\stackrel{<}{J}+\vec{R}$.

Subcase 2.2.2. If $g_{1}\left(\widehat{p}_{2}\right)>g_{2}\left(p_{+}\right)$, from the continuity we know that the Riemann solution is $\hat{R}+\stackrel{<}{J}+\vec{S}$.

Case $3\left(p_{-} \tau_{-}^{\gamma}>p_{+} \tau_{+}^{\gamma}\right)$.

Subcase $3.1\left(u_{-} \leq f_{2}(0)\right.$ (see Figure 4$\left.)\right)$. It is obvious that there exists a point $\widehat{p}_{3} \in\left(p_{*}, p_{-}\right)$such that $f_{1}\left(\widehat{p}_{3}\right)=f_{2}(0)$ and $g_{1}\left(\widehat{p}_{3}\right)>g_{2}(0)$. And we get the Riemann solution $\vec{R}+\vec{J}+\vec{R}$.

Subcase $3.2\left(u_{-}>f_{2}(0)\right.$ (see Figure 5$\left.)\right)$. Since there exists a point $\widehat{p}_{4} \in\left(0, p_{*}\right)$ such that $f_{2}\left(\hat{p}_{4}\right)=u_{-}$, we divide it into two subcases.

Subcase 3.2.1. If $g_{1}\left(p_{-}\right) \geq g_{2}\left(\widehat{p}_{4}\right)$, the Riemann solution is $\grave{R}+\vec{J}+\vec{R}$.

Subcase 3.2.2. If $g_{1}\left(p_{-}\right)<g_{2}\left(\widehat{p}_{4}\right)$, the Riemann solution is $\overleftarrow{S}+\vec{J}+\vec{R}$. [2].

From the above discussions, we have the result as follows

Theorem 1. There exists uniquely piecewise smooth solution to the Riemann problem (3) and (4). 


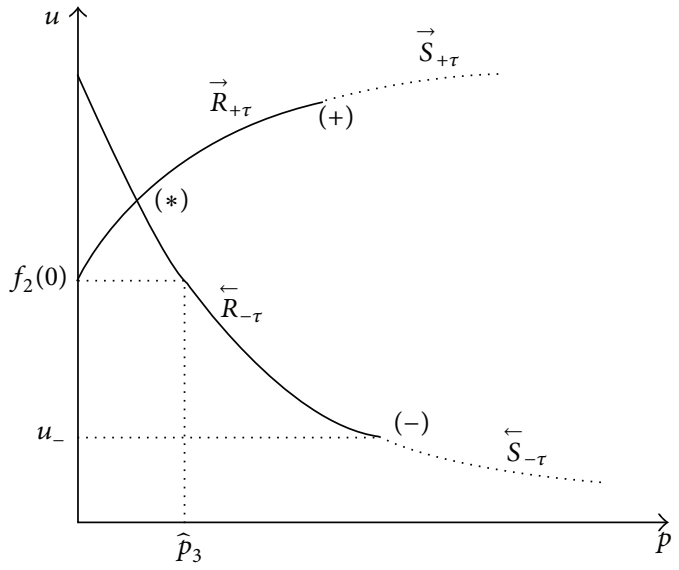

FIGURE 4: $f_{1}\left(p_{-}\right)=u_{-} \leq f_{2}(0)$.

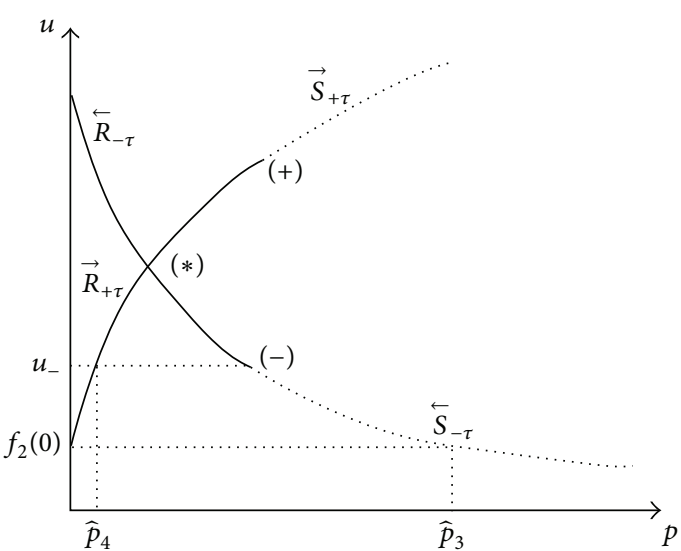

FIGURE 5: $f_{1}\left(p_{-}\right)=u_{-}>f_{2}(0)$.

\section{Solutions of the Generalized Riemann Problem (3) and (6)}

In what follows, we investigate the solutions for the discontinuous initial value problem (3) with (6) in a neighborhood of the origin $(t>0)$ on the $(x, t)$ plane. From the results in [12], the classical solution $\left(\tau_{l}, p_{l}, u_{l}\right)(x, t)\left(\left(\tau_{r}, p_{r}, u_{r}\right)(x, t)\right)$ can be defined in a strip domain $D_{l}\left(D_{r}\right)$ for a local time. The right boundary of $D_{l}$ has characteristic $O A: x=\lambda_{-} t$, and the left boundary of $D_{r}$ has characteristic $O B: x=\lambda_{+} t$ (see Figure 6).

According to the different cases of the corresponding Riemann solutions of (3) and (4), we construct the solutions case by case for (3) with (6). For simplicity, we only consider some interesting phenomena. For the other cases, a similar discussion to that of the generalized Riemann problem (3) and (6) can be carried out, and we omit them. For simplicity, we use the same symbols after perturbation since there is not any confusion.

Case 1 (when $p_{-} \tau_{-}^{\gamma}=p_{+} \tau_{+}^{\gamma}$, the corresponding Riemann solution is $\stackrel{R}{R} \vec{R}$ ). After perturbation, we obtain two subcases

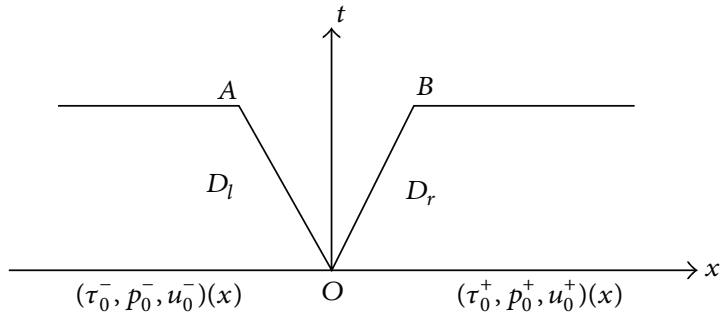

FIGURE 6: The discussed region.

which are that $p_{-} \tau_{-}^{\gamma}<p_{+} \tau_{+}^{\gamma}$ or $p_{-} \tau_{-}^{\gamma}>p_{+} \tau_{+}^{\gamma}$. In what follows, we discuss our problem in two subcases.

Subcase $1.1\left(p_{-} \tau_{-}^{\gamma}<p_{+} \tau_{+}^{\gamma}\right)$.

Subcase 1.1.1. If $f_{1}(0) \leq u_{+}$or $f_{1}(0)>u_{+}$and $g_{1}\left(\widehat{p}_{2}\right) \leq g_{2}\left(p_{+}\right)$ after perturbation, we obtain that the perturbed Riemann solution is $\overleftarrow{R}+\stackrel{\zeta}{J}+\vec{R}$. For this subcase, the perturbation can not affect the corresponding Riemann solution which shows that the corresponding Riemann solution is stable.

Subcase 1.1.2. If $f_{1}(0)>u_{+}$and $g_{1}\left(\hat{p}_{2}\right)>g_{2}\left(p_{+}\right)$after perturbation, we obtain that the perturbed Riemann solution is $\overleftarrow{R}+\grave{J}+\vec{S}$. For this subcase, the perturbation can affect the corresponding Riemann solution which shows that the corresponding Riemann solution is unstable.

Subcase $1.2\left(p_{-} \tau_{-}^{\gamma}>p_{+} \tau_{+}^{\gamma}\right)$.

Subcase 1.2.1. If $u_{-} \leq f_{2}(0)$ or $u_{-}>f_{2}(0)$ and $g_{1}\left(p_{-}\right) \geq g_{2}\left(\hat{p}_{4}\right)$ after perturbation, it follows that the perturbed Riemann solution is $\stackrel{\leftarrow}{R}+\vec{J}+\vec{R}$.

Subcase 1.2.2. If $u_{-}>f_{2}(0)$ and $g_{1}\left(p_{-}\right)<g_{2}\left(\hat{p}_{4}\right)$ after perturbation, it yields that the perturbed Riemann solution is $\overleftarrow{S}+\vec{J}+\vec{R}$.

Theorem 2. For Case 1 (see Figure 7), although there is no contact discontinuity of the corresponding Riemann solution, the contact discontinuity appears after perturbation. Furthermore, for Subcases 1.1.1 and 1.2.1, we observe that the corresponding Riemann solution is stable, while for Subcases 1.1.2 and 1.2.2, the backward (forward) rarefaction wave of the corresponding Riemann solution can be transformed into the backward (forward) shock wave after perturbation which shows that the structure of the Riemann solution is unstable.

Case $2\left(p_{-} \tau_{-}^{\gamma}<p_{+} \tau_{+}^{\gamma}\right)$. After perturbation, it still holds that $p_{-} \tau_{-}^{\gamma}<p_{+} \tau_{+}^{\gamma}$. In what follows, we discuss in detail our perturbed Riemann problem for this case.

Subcase 2.1. If $f_{1}(0) \leq u_{+}$, the corresponding Riemann

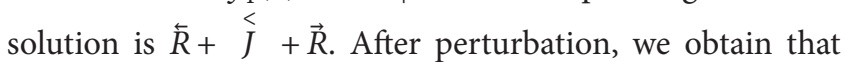
$f_{1}(0) \leq u_{+}$or $f_{1}(0)>u_{+}$; we construct the perturbed Riemann solutions as follows. 


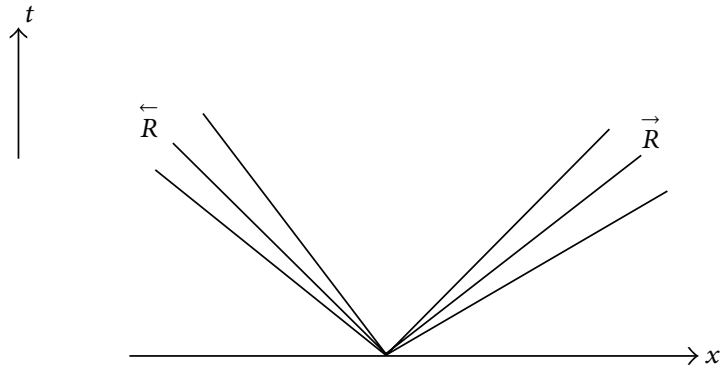

(a) The Riemann solution

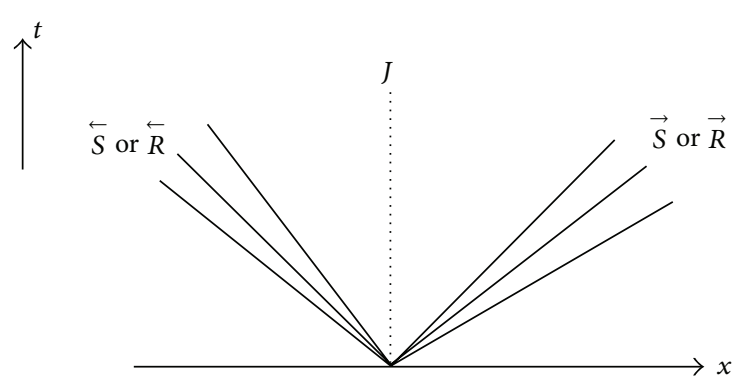

(b) The perturbed solution in the neighborhood of the origin $(t>$ 0)

Figure 7: Solutions in Case 1.

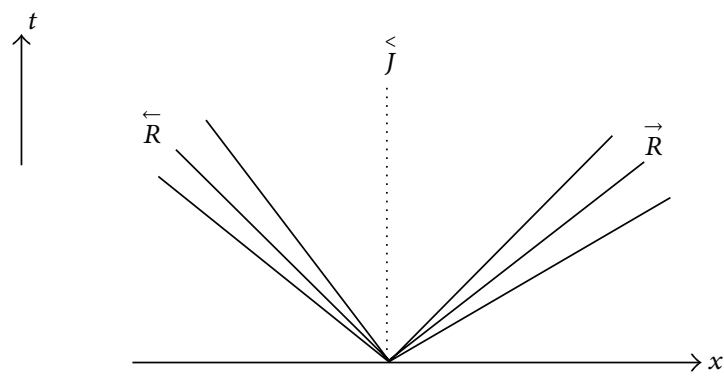

(a) A perturbed solution in the neighborhood of the origin $(t>$ $0)$

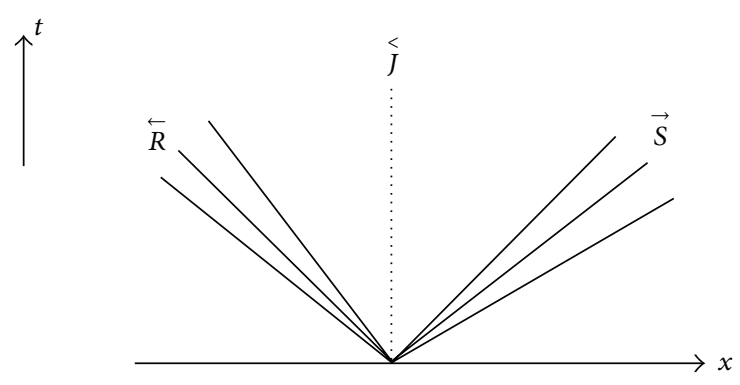

(b) A perturbed solution in the neighborhood of the origin $(t>0)$

FIGURE 8: Solutions in Case 2.

Subcase 2.1.1. When $f_{1}(0) \leq u_{+}$or $f_{1}(0)>u_{+}$and $g_{1}\left(\widehat{p}_{2}\right) \leq$ $g_{2}\left(p_{+}\right)$after perturbation, we know the perturbed Riemann solution is still $\overleftarrow{R}+\stackrel{<}{J}+\vec{R}$.

Subcase 2.1.2. When $f_{1}(0)>u_{+}$and $g_{1}\left(\widehat{p}_{2}\right)>g_{2}\left(p_{+}\right)$after perturbation, the perturbed Riemann solution is $\overleftarrow{R}+\vec{J}+\vec{S}$.

Subcase 2.2. If $f_{1}(0)>u_{+}$and $g_{1}\left(\widehat{p}_{2}\right) \leq g_{2}\left(p_{+}\right)$, the corresponding Riemann solution is $\overleftarrow{R}+\stackrel{<}{J}+\vec{R}$. After perturbation, we have $f_{1}(0)>u_{+}, g_{1}\left(\hat{p}_{2}\right) \leq g_{2}\left(p_{+}\right)$, or $g_{1}\left(\hat{p}_{2}\right)>g_{2}\left(p_{+}\right)$, we construct the perturbed Riemann solutions as follows.

Subcase 2.2.1. When $f_{1}(0)>u_{+}$and $g_{1}\left(\widehat{p}_{2}\right) \leq g_{2}\left(p_{+}\right)$after perturbation, we obtain that the perturbed Riemann solution is still $\overleftarrow{R}+\stackrel{<}{J}+\vec{R}$.

Subcase 2.2.2. When $f_{1}(0)>u_{+}$and $g_{1}\left(\widehat{p}_{2}\right)>g_{2}\left(p_{+}\right)$after

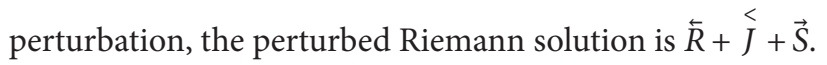

Subcase 2.3. If $f_{1}(0)>u_{+}$and $g_{1}\left(\widehat{p}_{2}\right)>g_{2}\left(p_{+}\right)$, the corresponding Riemann solution is $\overleftarrow{R}+\stackrel{<}{J}+\vec{S}$. After perturbation, we obtain that $f_{1}(0)>u_{+}$and $g_{1}\left(\widehat{p}_{2}\right)>g_{2}\left(p_{+}\right)$still hold and it follows that the perturbed Riemann solution is $\overleftarrow{R}+\stackrel{<}{J}+\vec{S}$.

Theorem 3. For Subcases 2.1.1, 2.2.1, and 2.3 (see Figure 8), the corresponding Riemann solution remains unchanged after perturbation which shows that the corresponding Riemann solution is stable and the perturbation can not affect the corresponding Riemann solution. While, for Subcases 2.1.2 and 2.2.2, the forward rarefaction wave of the corresponding Riemann solution can be transformed into the forward shock wave after perturbation which shows that the corresponding Riemann solution is unstable.

Case $3\left(p_{-} \tau_{-}^{\gamma}>p_{+} \tau_{+}^{\gamma}\right)$. After perturbation, it still holds that $p_{-} \tau_{-}^{\gamma}>p_{+} \tau_{+}^{\gamma}$.

Subcase 3.1. If $u_{-} \leq f_{2}(0)$, the corresponding Riemann solution is $\vec{R}+\vec{J}+\vec{R}$. After perturbation, we obtain that $u_{-} \leq$ $f_{2}(0)$ or $u_{-}>f_{2}(0)$; we construct the perturbed Riemann solutions as follows.

Subcase 3.1.1. When $u_{-} \leq f_{2}(0)$ or $u_{-}>f_{2}(0)$ and $g_{1}\left(p_{-}\right) \geq$ $g_{2}\left(\widehat{p}_{4}\right)$ after perturbation, we know the perturbed Riemann solution is still $\overleftarrow{R}+\vec{J}+\vec{R}$.

Subcase 3.1.2. When $u_{-}>f_{2}(0)$ and $g_{1}\left(p_{-}\right)<g_{2}\left(\hat{p}_{4}\right)$ after perturbation, the perturbed Riemann solution is $\overleftarrow{S}+\vec{J}+\vec{R}$.

Subcase 3.2. If $u_{-}>f_{2}(0)$ and $g_{1}\left(p_{-}\right) \geq g_{2}\left(\widehat{p}_{4}\right)$, the corresponding Riemann solution is $\vec{R}+\vec{J}+\vec{R}$. After perturbation, we have $u_{-}>f_{2}(0), g_{1}\left(p_{-}\right) \geq g_{2}\left(\widehat{p}_{4}\right)$, or $g_{1}\left(p_{-}\right)<g_{2}\left(\widehat{p}_{4}\right)$; we construct the perturbed Riemann solutions as follows. 


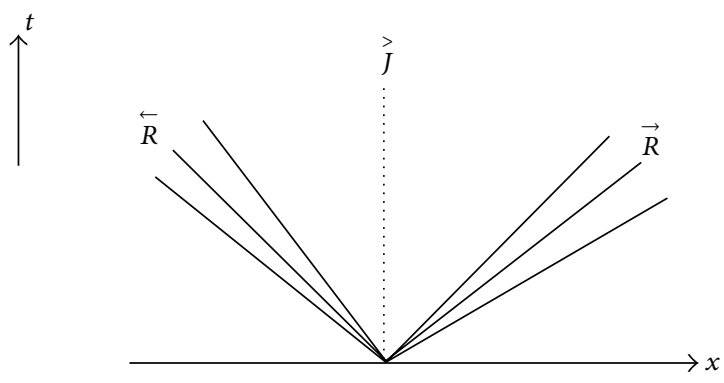

(a) A perturbed solution in the neighborhood of the origin $(t>$

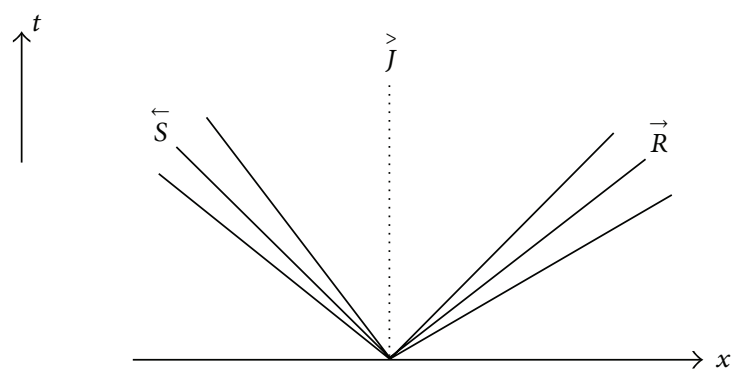

(b) A perturbed solution in the neighborhood of the origin $(t>0)$

Figure 9: Solutions in Case 3.

Subcase 3.2.1. When $u_{-}>f_{2}(0)$ and $g_{1}\left(p_{-}\right) \geq g_{2}\left(\widehat{p}_{4}\right)$ after perturbation, we obtain that the perturbed Riemann solution is still $\hat{R}+\vec{J}+\vec{R}$.

Subcase 3.2.2. When $u_{-}>f_{2}(0)$ and $g_{1}\left(p_{-}\right)<g_{2}\left(\widehat{p}_{4}\right)$ after perturbation, the perturbed Riemann solution is $\overleftarrow{S}+\vec{J}+\vec{R}$.

Subcase 3.3. If $u_{-}>f_{2}(0)$ and $g_{1}\left(p_{-}\right)<g_{2}\left(\widehat{p}_{4}\right)$, the corresponding Riemann solution is $\hat{S}+\vec{J}+\vec{R}$. After perturbation, we find that $u_{-}>f_{2}(0)$ and $g_{1}\left(p_{-}\right)<g_{2}\left(\hat{p}_{4}\right)$ still hold and it follows that the perturbed Riemann solution is $\overleftarrow{S}+\vec{J}+\vec{R}$.

Theorem 4. For Subcases 3.1.1, 3.2.1, and 3.3 (see Figure 9), the corresponding Riemann solution remains unchanged after perturbation which shows that the corresponding Riemann solution is stable. While, for Subcases 3.1.2 and 3.2.2, the backward rarefaction wave of the corresponding Riemann solution can be transformed into the backward shock wave after perturbation which shows that the corresponding Riemann solution is unstable.

Based on the above discussions, we summarize our main result as follows.

Theorem 5. There exists uniquely solution for the generalized Riemann problem (3) and (6) in a neighborhood of the origin $(t>0)$ in the $(x, t)$ plane. The contact discontinuity may appear after perturbation although there is no contact discontinuity in the corresponding Riemann solution. Furthermore, for most cases, the perturbed Riemann solutions of (3) and (6) are stable. While, for some few cases, the perturbed Riemann solutions of (3) and (6) are unstable under such local small perturbations of the Riemann data.

\section{Competing Interests}

The authors declare that they have no competing interests.

\section{Acknowledgments}

This work was partially supported by NSFC 11326156 and partially supported by the Foundation for Young Scholars of Shandong University of Technology.

\section{References}

[1] H. Cabannes, Theoretical Magnetofluid Dynamics, Academic Press, New York, NY, USA, 1970.

[2] Y. Hu and W. Sheng, "The Riemann problem of conservation laws in magnetogasdynamics," Communications on Pure and Applied Analysis, vol. 12, no. 2, pp. 755-769, 2013.

[3] W. R. Hu, Universe Magnetogasdynamics, Chinese Series, Science Press, Beijing, China, 1987.

[4] W. X. Li, One-Dimensional Unsteady Flow and Shock Wave, National Defence Industrial Press, Beijing, China, 2003.

[5] T. Raja Sekhar and V. D. Sharma, "Riemann problem and elementary wave interactions in isentropic magnetogasdynamics," Nonlinear Analysis: Real World Applications, vol. 11, no. 2, pp. 619-636, 2010.

[6] C. Shen, "The limits of Riemann solutions to the isentropic magnetogasdynamics," Applied Mathematics Letters, vol. 24, no. 7, pp. 1124-1129, 2011.

[7] Y. Liu and W. Sun, "Riemann problem and wave interactions in magnetogasdynamics," Journal of Mathematical Analysis and Applications, vol. 397, no. 2, pp. 454-466, 2013.

[8] F. G. Liu, "Life-span of classical solutions for one-dimensional hydromagnetic flow," Applied Mathematics and Mechanics, vol. 28, no. 4, pp. 511-520, 2007.

[9] M. Ben-Artzi, "The generalized Riemann problem for reactive flows," Journal of Computational Physics, vol. 81, no. 1, pp. 70101, 1989.

[10] T. Chang and L. Hsiao, The Riemann Problem and Interaction of Waves in Gas Dynamics, vol. 41 of Pitman Monographs, Longman Scientific and Technical, Essex, UK, 1989.

[11] T. T. Li, Global Classical Solutions for Quasilinear Hyperbolic Systems, vol. 32, John Wiley \& Sons, New York, NY, USA, 1994.

[12] T. T. Li and W. C. Yu, Boundary Value Problems for Quasilinear Hyperbolic Systems, Duke University Mathematics, 1985.

[13] Y.-J. Liu and W.-C. Sheng, "Generalized Riemann problem for gas dynamic combustion," Applied Mathematics and Mechanics, vol. 32, no. 8, pp. 1079-1090, 2011.

[14] W. Sheng, M. Sun, and T. Zhang, “The generalized Riemann problem for a scalar nonconvex Chapman-Jouguet combustion model," SIAM Journal on Applied Mathematics, vol. 68, no. 2, pp. 544-561, 2007. 


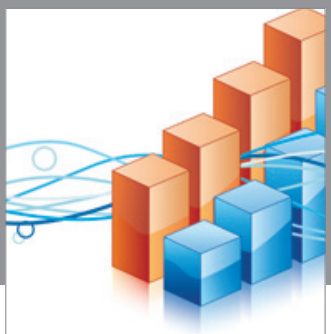

Advances in

Operations Research

vatem alat4

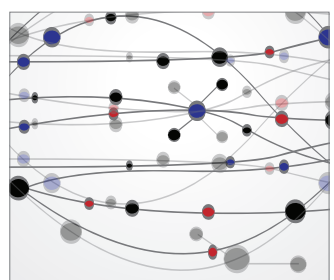

\section{The Scientific} World Journal
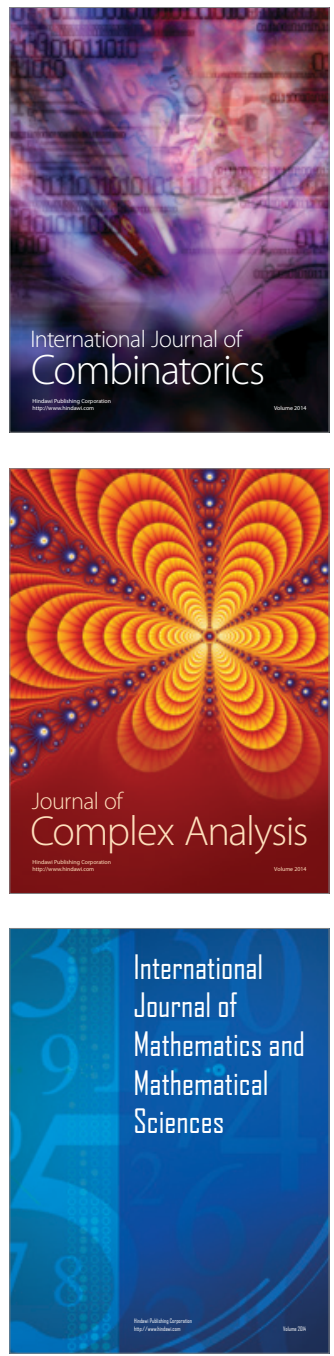
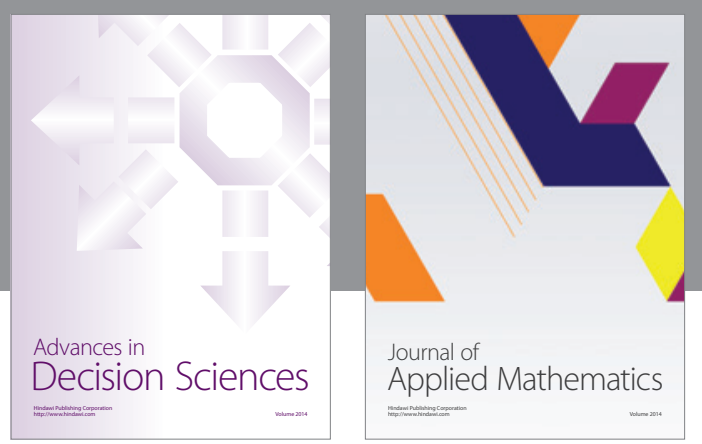

Algebra

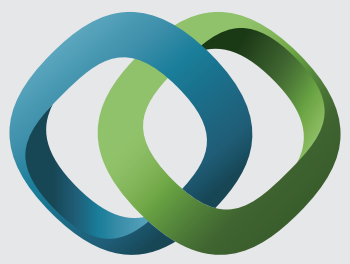

\section{Hindawi}

Submit your manuscripts at

http://www.hindawi.com
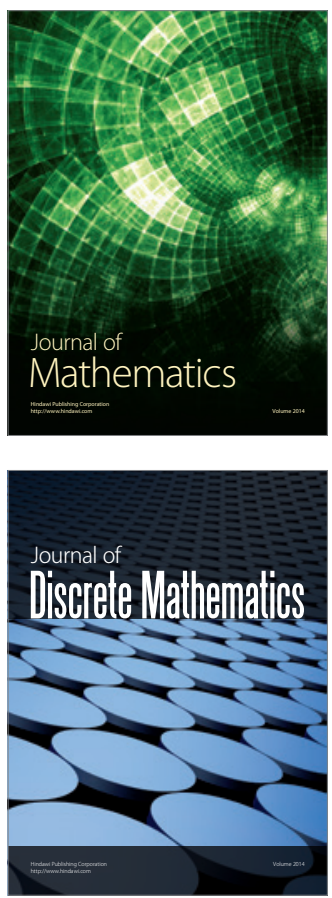

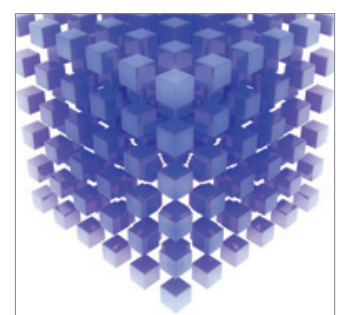

Mathematical Problems in Engineering
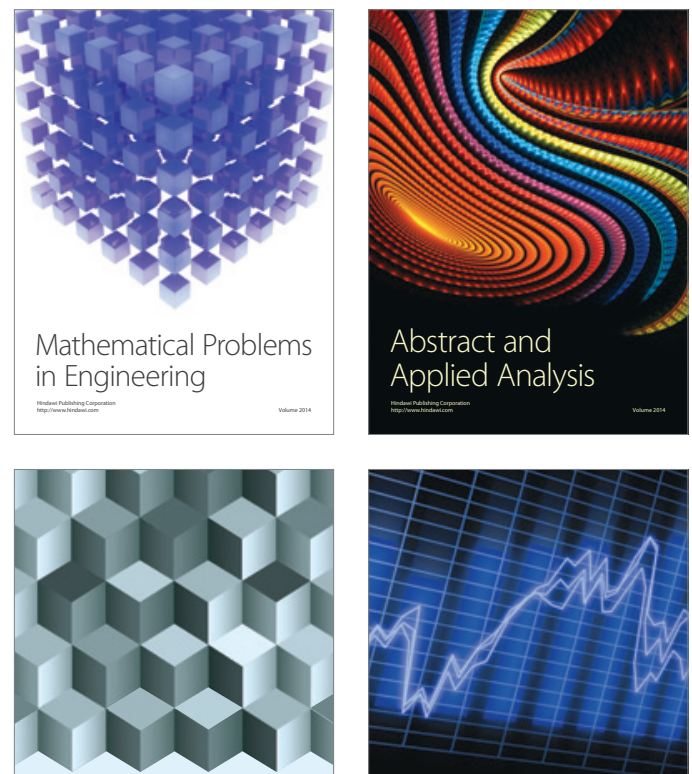

Journal of

Function Spaces

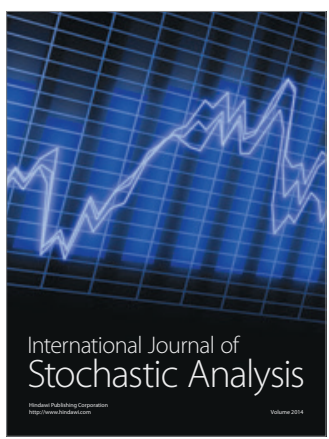

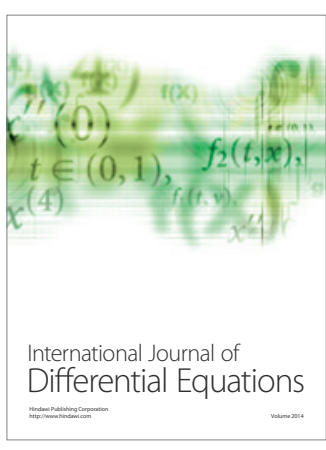
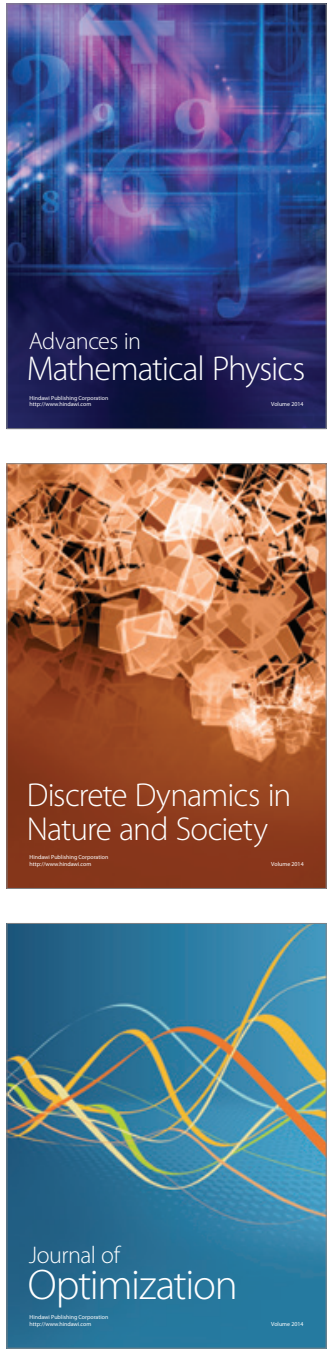